\title{
Lumen
}

Selected Proceedings from the Canadian Society for Eighteenth-Century Studies

\section{Temps social et temps personnel dans Les Égarements de Crébillon, ou la durée au risque du récit}

\section{Jacques Wagner}

Volume 18, 1999

Representations of Time in the XVIIIth Century

Le temps et ses représentations au dix-huitième siècle

URI : https://id.erudit.org/iderudit/1012375ar

DOI : https://doi.org/10.7202/1012375ar

Aller au sommaire du numéro

Éditeur(s)

Canadian Society for Eighteenth-Century Studies / Société canadienne d'étude du dix-huitième siècle

ISSN

1209-3696 (imprimé)

1927-8284 (numérique)

Découvrir la revue

Citer cet article

Wagner, J. (1999). Temps social et temps personnel dans Les Égarements de Crébillon, ou la durée au risque du récit. Lumen, 18, 185-200.

https://doi.org/10.7202/1012375ar

Copyright (c) Canadian Society for Eighteenth-Century Studies / Sociéte canadienne d'étude du dix-huitième siècle, 1999
Ce document est protégé par la loi sur le droit d'auteur. L'utilisation des services d'Érudit (y compris la reproduction) est assujettie à sa politique d'utilisation que vous pouvez consulter en ligne.

https://apropos.erudit.org/fr/usagers/politique-dutilisation/ 


\section{Temps social et temps personnel dans Les Égarements de Crébillon, ou la durée au risque du récit}

Le temps est un marmot qui joue au

tric-trac; son règne est d'un marmot.

Héraclite, Fragments ${ }^{1}$

Le temps n'est probablement pas la catégorie a priori de l'expérience la plus appréciée dans la culture de l'Ancien Régime; son augustinisme durable et son penchant stoïcien avaient goûté et accepté les mépris antiques formulés à l'encontre de la dimension temporelle de l'existence humaine: '[s]'élever au-dessus de la terre, c'est mériter les étoiles', déclamait la philosophie consolatrice dans la prison de Boèce, ${ }^{2}$ ni le bien ni le vrai ne passaient pour compatibles avec les flux et les horizons illusoires du temps; ${ }^{3}$ Pascal, La Rochefoucauld, chacun, dans son style, reprendra cette antienne dédaigneuse, ${ }^{4}$ plus tard et plus systématiquement, JeanJacques Rousseau, à la recherche d'un 'état où l'âme trouve une assiette assez solide pour s'y reposer tout entière et rassembler là tout son être, sans avoir besoin de rappeler le passé ni d'enjamber sur l'avenir, où le présent dure toujours sans néanmoins marquer sa durée et sans aucune trace de succession' orchestrera le thème en une lamentation lyrique et philosophique à la fois:

le bonheur que mon coeur regrette n'est point composé d'instants fugitifs mais un état simple et permanent, qui n'a rien de vif en lui-même mais dont la durée accroît le charme au point d'y trouver enfin la suprême félicité. Tout est dans un flux continuel sur la terre: rien n'y garde une forme constante et arrêtée, et nos affections qui s'attachent aux choses extérieures passent et changent nécessairement comme elles. ${ }^{5}$

Rousseau indique avec précision la forme que doit prendre le temps pour acquérir une valeur aux yeux des moralistes classiques: il doit s'écarter du point (crise ou acmée) et se rapprocher le plus possible de la durée plane (continuum tragique ou extatique). ${ }^{6}$ Or l'une des caractéristiques des romans de Crébillon ne serait-elle pas précisément son refus ou son 
incapacité à inscrire ses personnages dans une durée ferme et porteuse de sens? Qu'il s'agisse du temps social des activités mondaines (réceptions, promenades, jeux, repas, conversations) et des responsabilités morales (éducation familiale, par exemple) ou du temps personnel des activités individuelles et des manières de vivre quotidiennes (humeurs, comportements, désirs, méditations, rêveries et projets propres), le roman de Crébillon semble multiplier les discordances et les incompatibilités entre les deux catégories temporelles autant qu'à l'intérieur de chaque type. De sorte que nous explorerons deux aspects du temps dans les Egarements du coeur et de l'esprit: la durée interdite dans les relations sociales et la défaite du temps intérieur. Enfin, nous évoquerons un troisième aspect: le temps rédactionnel du mémorialiste.

\section{L'impossible durée des relations sociales}

Dans ce roman, le temps s'impose à la conscience des personnages de façon impérieuse, pathétique ou burlesque. Le mémorialiste laisse place à des remarques cruelles sur les effets et les méfaits de l'âge, sur la corruption des temps présents et la supériorité des temps anciens.

La description du passé insiste sur son excellence disparue par incapacité morale des contemporains; ses caractéristiques se résument en deux mots: durée - qui implique continuité, fidélité - et lenteur ${ }^{7}$ qui comporte respect, sagesse; en qualité et en quantité, les temps modernes ne sauraient rivaliser avec les temps anciens:

Si nous en croyons d'anciens Mémoires, les femmes étaient autrefois plus flattées $\mathrm{d}$ 'inspirer le respect que le désir, et peut-être y gagnaient-elles. A la vérité, on leur parlait d'amour moins promptement, mais celui qu'elles faisaient naître n'en était que plus satisfaisant, et que plus durable. ${ }^{8}$ (19)

Il arrive toutefois que dans les temps présents, les individus les plus corrompus recourent parfois à la lenteur: Mme de Lursay et Mme de Senanges, par exemple; mais l'une et l'autre le font de manière tactique, par intelligence rusée; Mme de Lursay pour faire croire à Meilcour qu'il vit une histoire d'amour à l'ancienne: '[c]e n'est pas qu'elle voulût retarder longtemps l'aveu de sa faiblesse. Huit jours pour cet article seulement suffisaient à sa vertu [...] . L'amour qu'elle avait pour moi l'engageait à ce manège' (39); Mme de Senanges pour appliquer une technique de séduction qui dicte d'affecter une méprisante indifférence, 'uniquement pour qu'on fût tenté d'en triompher': '[c]e fut apparemment pour se conformer à cette sage maxime, qu'elle ne commença à m'être favorable qu'une heure environ après m'avoir vu' (195). La lenteur moderne n'est rien d'autre qu'un artifice de l'intelligence égo- 
centrique à la recherche du plaisir ou d'une vengeance. Mme de Lursay impose des 'gradations' à son jeune amant pour son profit autant qu'elle sait jouer du temps au détriment des autres, si nécessaire: 'patiente dans ses vengeances comme dans ses piaisirs, elle savait les attendre du temps, lorsque le moment ne les lui fournissait pas' (23); car les temps présents préféreraient la vitesse (privée de scrupule), comme le souhaite Meilcour au moment de rompre avec Lursay: '[l]e parti que je lui voyais prendre était donc le seul qui pût me convenir; il nous faisait rompre sans éclat, sans altercations, sans lenteurs' (254); et l'instantané (privé de conscience), comme le rappelle le mémorialiste: '[l]a première vue décidait une affaire, mais en même temps il était rare que le lendemain la vît subsister' (19); une jeune femme, non seulement mépriserait la durée morale du coeur: '[l]e mérite de s'attacher un amant pour toujours ne vaut pas à ses yeux celui d'en enchaîner plusieurs' (40), mais dépendrait du temps aléatoire du corps: 'une jolie femme dépend moins d'ellemême que des circonstances, et par malheur il s'en trouve tant, de si peu prévues, de si pressantes, qu'il n'y a point à s'étonner si, après plusieurs aventures, elle n'a connu ni l'amour, ni son coeur' (ibid.). Versac prendra le relais du mémorialiste quand il tentera de faire la leçon à Meilcour:

[les femmes] ne regardent jamais comme vraies passions que celles qui commencent par les enlever à elles-mêmes. Ces attachements, que l'habitude de se voir forme quelquefois, ne leur paraissent presque toujours que des affaires de convenance, dont elles ne croient devoir s'occuper que médiocrement. L'impression qu'on ne leur fait qu'avec lenteur, n'agit jamais sur elle avec vivacité. (243)

Ce rythme heurté du sentiment correspond à une manière commune et sociale de vivre. Versac développe devant Meilcour une théorie des ridicules articulée sur les variations temporelles du 'crédit': les ridicules en crédit 'qui, dépendant du caprice, sont sujets à varier, n'ont, comme toutes les modes, qu'un certain temps pour plaire, et qui, pendant qu'ils sont en règne, effacent tous les autres. C'est dans le temps de leur vogue qu'il faut les saisir' (232). Elle s'étend même à la raison: '[c]'est presque toujours à ceux d'entre nous qui raisonnent le plus profondément que l'on doit ces opinions absurdes qui font honte à l'esprit, et ce maintien affecté qui gâte et contraint la figure' (233); et au langage - il ne convient pas de prendre au sérieux les désignations linguistiques de la réalité qui varient elles-aussi au gré des modes:

Ne soyez point, au reste, arrêté par le nom que je donne aux choses qui sont en possession de séduire: tant qu'un ridicule plaît, il est grâce, agrément, esprit, et ce n'est que quand, pour l'avoir usé, on s'en lasse, qu'on lui donne le nom qu'en effet il mérite. (ibid.) 
Le langage perd sa dimension monumentale de mémoire collective des significations, de répertoire commun des sens, de trésor partagé des signes. Le discours lui-même est atteint par cette évolution malheureuse pour une conscience classique. Versac se sent obligé, au moment où il projette de poursuivre sa leçon, de remettre 'à un autre jour' la suite: '[l]a conversation que nous venons d'avoir a été d'une longueur si énorme qu'avec plus d'ordre, et des idées plus approfondies, elle pourrait presque passer pour un Traité de morale' (250). La longueur est une faute de goût dans une société qui ne supporte plus un développement rationnel des idées: '[e]st-ce que l'on peut, sans s'appesantir, suivre une idée? On peut la proposer, mais a-t-on jamais le temps de l'établir? N'est-ce pas même blesser la bienséance que d'y songer? Oui. La conversation, pour être vive, ne saurait être assez peu suivie' (248); une règle 'bizarre' (249) de la bonne conversation impose l'interruption systématique et rapide, qui permet une succession heurtée de sujets les moins compatibles abordés par des locuteurs les plus contrastés: ainsi 'quelqu'un qui parle guerre se laisse interrompre par une femme qui veut parler sentiment; que celle-ci, au milieu de toutes les idées que lui fait naître un sujet si noble, et qu'elle possède si bien, se taise pour écouter un couplet galamment obscène' (248). La durée aurait ainsi perdu toute vertu, si l'on peut dire, aux yeux de la société moderne vouée au changement, au caprice, à la mode, à la vitesse et à la brièveté, comme si elle se confondait désormais avec le malaise ou même le malheur.

C'est bien ce qu'éprouvent à leur détriment les personnages qui s'y trouvent confrontés. Le premier cas est celui de l'âge. Il est évoqué à plusieurs reprises de façon négative soit parce qu'il révèle les variations de comportement des êtres humains, surtout chez les femmes (40-41), parfois chez les hommes (199); ${ }^{9}$ soit parce qu'il suscite une régulation, contrôlée par la société, des sentiments personnels, comme le déclare Mme de Senanges: '[i]l est un âge où l'on sait qu'il faut se rendre justice' (201) pour éviter de se perdre 'à jamais dans le monde' (203), car selon le mot de Versac 'on ne souffre pas tranquillement' qu'une femme qui ne serait plus 'de la première jeunesse' cherchât à inspirer ou à faire naître des sentiments (201); Mme de Lursay, parfaitement informée des moeurs de la bonne société, ne pense pas différemment et avoue à Meilcour son souci de les respecter: '[n]aturellement je suis peu susceptible, et pour me sauver des égarements de la jeunessse, je n'ai pas eu besoin de réfléchir. Il me paraîtrait d'un extrême ridicule de donner aujourd'hui dans un travers qui, par mille raisons que vous ne sentez pas, pourrait m'être moins pardonné que jamais' (86); c'est en outre une raison de moquerie cruelle: '[q]uelle horreur! Madame de ${ }^{* *}$ ! Mais cela a cent ans!', s'indigne Mme de Senanges en apprenant que Pranzi 'allait souper en tête-à-tête avec la vieille Mme de ${ }^{* * * \prime}$ (138); elle-même subit l'humeur 
sardonique du jeune Meilcour en visite chez elle: '[e]lle me parut comme la veille à peu près, si ce n'est qu'au grand jour je lui trouvai quelques années de plus et quelques beautés de moins' (187). Versac se révèle plus méchant encore:

[m]oi, par exemple, je connais des femmes qui ont vieilli beaucoup, extrêmement, qui par conséquent sont devenues laides et ne s'en doutent seulement pas; et qui croient, de la meilleure foi du monde, avoir encore tous les charmes de la jeunesse parce qu'elles en ont conservé soigneusement tous les travers. (202)

C'est aussi une manière de rejeter dans un passé démodé: '[e]lle a peut-être plu jadis, mais personne d'aujourd'hui n'était de ce temps-là' (199); enfin, l'âge est un élément discursif efficace dans une argumentation qui cherche à dénigrer: Versac soutient que Mme de Lursay a plus de quarante ans parce qu'il ne l'aime 'pas assez pour permettre qu'elle n'ait que son âge' (202).

Ni la durée biologique ni la durée sentimentale ne trouvent grâce aux yeux d'une société mondaine tout entière occupée de l'instant présent; elles séparent, elles excluent, elles embarrassent aussi les individus eux-mêmes. Meilcour ressent son éducation comme un passé pesant qui l'enferme dans une attitude inadaptée au projet de rompre avec Lursay: '[t]out déterminé que j'étais à rompre avec elle, je ne savais pas comment lui dire que je ne l'aimais plus. Le respect qu'elle m'avait inspiré était en moi comme ces préjugés d'enfance, contre lesquels on se révolte longtemps avant que de pouvoir les détruire' (254); plus intimement, la durée est redoutée en ce qu'elle inscrirait dans l'âme des sensations qui, y persistant au-delà du moment de leur naissance, se heurteraient à la différence inattendue d'un instant ultérieur et provoqueraient de la sorte un sentiment de gêne: '[q]uelques choses que j'en pensasse dans ce moment, l'estime que $j$ 'avais eue pour elle me tyrannisait encore et me forçait à lui déguiser mes sentiments' (254).

La durée n'est quasiment jamais accompagnée de significations positives: la bonne comtesse qu'on ne voit plus dans les salons passerait vite pour s'être 'jetée dans la dévotion', même aux yeux de l'excellente Mme de Meilcour; or il n'en est rien; elle est seulement 'depuis huit jours' (111) dans un fatal délaissement à la suite d'une infidélité amoureuse; en sens inverse, Versac est en butte à des rumeurs déplaisantes pour lui d'engagement amoureux exclusif (206); Mme de Lursay est de même victime du qu'en dira-t-on: 'malgré l'air prude qu'elle avait pris, on s'obstinait toujours à la soupçonner' (22).

Enfin, le romancier enlève à ses personnages le bonheur de vivre le moindre moment d'entente cordiale ou amoureuse: chaque dialogue, chaque rencontre est l'occasion d'une dispute, d'un malentendu, d'une 
méprise, comme si la moindre durée se transformait en mésentente fatale. Le deuxième dialogue des amants en est un bon exemple: lancé par Mme de Lursay sur un sourire engageant, il évolue dans un sens agressif; d'abord des attaques véhémentes relatives au manque de savoir-vivre de Meilcour: '[m]ais qu'avez-vous donc? Pourquoi cet air froid dont vous m'accablez?'; puis des reproches accusateurs venus d'une femme soudain tremblante: '[s]avez-vous bien que votre taciturnité me fait peur?'; enfin, un brutal changement de sujet: '[m]ais à propos, m'aimez-vous toujours bien? Je crois que non', suivi d'une exclamation humiliante à la troisième personne: '[c]e pauvre Meilcour!'; celui-ci terrassé par ces coups redoublés au long d'une tirade sans pause ne peut qu'articuler une plainte faible: '[e]n est-ce assez, madame [...] et devriez-vous ajouter, à la façon dont vous recevez mes soins, des discours qui me tuent?' (43). Meilcour, conformément à son éducation, donne une teinte tragique à ce dialogue paradoxal; Crébillon est capable d'en composer aussi en style comique, surtout lorsque les interlocuteurs sont eux-mêmes des personnages bouffons, comme Senanges et Mongennes au jardin des Tuileries: leurs sentiments progressent des 'alarmes' aux 'insultes', leurs réponses s'échangent avec un 'air sérieux' de plus en plus 'sec' et 'aigre', leurs regards froids montrent qu'elles 'se tenaient pour brouillées' (196); et Meilcour conclut: '[e]t j'eus la gloire en commençant ma carrière, de désunir deux femmes auxquelles je ne pensais pas' (195); le dialogue se termine sur la même remarque que le précédent: le temps passé ensemble sépare; le présent, dès qu'il se prolonge au-delà de l'instant, serait-il un cadeau empoisonné? Il semble que ce soit toujours le cas.

Le moment partagé est en effet souvent un temps négatif: mésentente, malentendu, équivoque, méprise, timidité, défi, sarcasme, rêverie, incompréhension, vanité, crainte, mauvaise foi, toutes les catégories du faux sens et du contresens sont à l'œuvre dans le roman. Les exemples abondent; retenons trois cas typiques. Le premier concerne deux personnages; après de nombreux et tactiques replis, Mme de Lursay se laisse aller à un geste d'abandon tendre auquel Meilcour n'est pas disposé à répondre: '[j]amais elle ne m'avait mieux montré sa tendresse et jamais je n'y avais aussi mal répondu' (170); à cette forme de la distance voulue s'ajoutent les séquences de distance involontaire due à l'incompréhension par interprétation aberrante: 'sûr qu'elle me faisait la plus sanglante des railleries, je ne m'en déterminai que plus à rompre d'aussi cruelles chaînes' (44); ou due au manque d'audace: '[q]uoique ses façons n'eussent rien de sévère, que même ses yeux me parlassent le langage le plus doux, je ne me sentis pas la force de lui répondre' (32). Le second type met en scène trois personnages: Mme de Lursay se trompe en croyant que Meilcour est amoureux de Mme de Senanges; enfin, le troisième type 
touche quatre personnages au moins: le souper de la huitième soirée rassemble, chez Mme de Lursay, la compagnie la plus hétéroclite qui soit moralement et sentimentalement: les libertins (Versac, Senanges, Pranzi), les honnêtes gens (Mme de Théville et sa fille, Meilcour); avec eux se croisent, dans l'espace clos du salon, les trois dimensions du temps: le passé avec Pranzi qui 'avait été cher' à Mme de Lursay, le présent avec Meilcour qui, amoureux d'Hortense, est attendu par la maîtresse de maison, enfin l'avenir avec Versac qui se tourne vers Hortense et avec Senanges qui recherche Meilcour. Or ces trois temporalités ne s'articulent pas harmonieusement. Elles s'entrechoquent: le passé pour Mme de Lursay relève d'une méchanceté tramée par Versac dont 'les discours la faisaient frémir'; son retour sous la forme d'un ancien amant mis en présence de l'amant du jour produit une 'cruelle situation' qui 'la plongeait dans un chagrin que l'on remarquait visiblement et dans la dernière confusion' (137); le retour d'un tel passé à un tel moment déséquilibre le présent du personnage visé que le romancier s' acharneà maltraiter, en lui enlevant la possibilité d'une rémission: 'Versac ne s'aperçut pas plus tôt du succès des coups qu'il portait à Mme de Lursay qu'il résolut de les redoubler' (137-38). Pour Meilcour, témoin de cette scène, le temps se fracture, le présent s'annule et compromet l'avenir: '[j]e conclus sur-lechamp de son silence et de son air humilié que Pranzi était infailliblement un de mes prédécesseurs' (137). Le moment présent de la rencontre s'alourdit de tensions provoquées par une volonté diaboliquement efficace. ${ }^{10}$ Mais le présent se défait aussi pour la raison exactement inverse: l'échec, pour Versac, de sa volonté de séduire devant la résistance d'Hortense. En outre, il se corrompt sous les agissements de Senanges qui 'tout occupée qu'elle était de son esprit, me faisait des agaceries sans ménagement', et de Meilcour lui-même qui en profite pour se venger $d$ 'Hortense 'que j'affectais alors de regarder avec autant d'indifférence quej'avais cru lui en remarquer pour moi' (144). Enfin, le présent coïncide avec un moment de malaise parce qu'il est opaque: ${ }^{11}$

dans l'état où je me trouvais, tout était pour moi matière à soupçon, tout augmentait mon inquiétude. Je voulais qu'elle me tînt compte d'un amour qu'elle n'avait pas dû pénétrer, il me semblait qu'elle ne pouvait pas se tromper aux mouvements qu'elle me faisait éprouver, que mon embarras et mes regards lui disaient assez combien elle m'avait rendu sensible, et qu'enfin j'aurais été entendu, si j'avais dû être aimé. (174)

Meilcour éprouve parfois cette opacité comme une douleur pénible:

Je n'entrevoyais rien qui pût me conduire sûrement à l'éclaircissement que je souhaitais, et m'aider à découvrir si Germeuil était cet inconnu que je savais 
aimé, ou si je n'avais pas à craindre quelque autre que lui. Absorbé dans cette confusion d'idées et de sentiments, les parcourant toutes, les éprouvant tous, sans m'arrêter sur aucun, je marchais auprès d'Hortense dans un état peu différent du sien. Je voulais interrompre sa rêverie et ne trouvai rien à lui dire. (219)

La ressemblance ne rassemble pas, elle isole. Le silence subi et l'impuissance à deviner le chiffre clair de l'existence caractérisent des personnages qui, poussés pourtant à 'se voir' tous les jours par une sociabilité impérieuse, ${ }^{12}$ ne s'entendent pas en réalité. Ironiquement, le romancier met dans la bouche de Lursay une remarque adressée à Meilcour qui s'appliquerait aussi bien à elle et conviendrait à la plupart des personnages: '[v]ous m'entendez mal' (168). Séparés par les mots, séparés par leurs désirs, séparés par leur morale, ils ne communient que dans la médisance, 'ressource' sans laquelle 'on soutient difficilement la conversation' (152).

Hors le jeu qui s'y substitue souvent pour maintenir un lien entre eux, les personnages sont victimes de la rêverie qui les isole des autres par l'indifférence qu'ils affichent à travers elle; elle présente deux versions, une forte et une faible, selon qu'elle isole longuement et profondément ou non, et trois degrés. Mme de Lursay illustre la version faible de la rêverie; placée 'dans une circonstance délicate', elle s'interroge sur la meilleure manière de réagir: 'elle demeura quelque temps rêveuse et sans parler' (124). La suspension prélude ici à une reprise de contact: 'elle prit enfin son parti. Ce fut de me témoigner, avec tendresse et dignité, qu'elle trouvait mes procédés extrêmement ridicules' (ibid.); rêverie de perplexité qui isole moins qu'elle ne relance l'action. Meilcour connaît aussi une rêverie de ce genre, mais un peu plus intérieure; sa perplexité le replie sur lui-même: '[s]ans pénétrer la cause du trouble qui se répandait dans mon âme, je m'y plongeai tout entier: je devins rêveur' (255). Enfin, la rêverie sépare totalement; c'est le cas d'Hortense: '[r]êveuse et comme embarrassée de ma présence, elle ne jetait sur moi que des regards tristes ou distraits' (128); durant le souper du huitième soir, 'elle tomba dans une rêverie profonde [...] et me regardait avec une sorte de mépris que je n'interprétais pas en bien, et dont de moment en moment je lui voulais plus de mal' (145). Meilcour en est lui aussi une victime fréquente que Mme de Lursay est obligée un jour de rappeler à ses devoirs de civilité: '[y] songez-vous? me dit-elle fort bas. Que voulezvous qu' on pense ici de la mine que vous faites? Il semble à vous voir que vous y soyez malgré vous' (183). Mme de Lursay perçoit bien le sens asocial de la rêverie; Meilcour l'associe à l'indifférence: '[i]nattentif et rêveur, à peine daignais-je répondre aux interrogations fréquentes dont 
elle ne cessait de me fatiguer' (198). La rêverie éloigne donc le personnage du moment présent de la communauté, s'oppose au temps social.

Cette impossibilité à vivre le présent comme un moment de bonheur fait de présence et de don, de connivence et de bonté ${ }^{13}$ vient d'un mélange de méchanceté joueuse, de narcissisme exacerbé, ${ }^{14}$ et aussi $d^{\prime}$ 'une peur de s'engager dans l'avenir. Sans parler de Versac qui s'efforce de réduire l'amour à une technique censée lui garantir, par avance, le résultat attendu en supprimant les risques inhérents à tout projet ${ }_{1}^{15}$ la pensée de l'avenir parasite la liberté d'action des personnages; soit de manière factice, tactique et conquérante - Mme de Lursay recourt à un argument digne des Précieuses quand elle prétend devant Meilcour, probablement sensible à ce genre de délicatesse du fait de son éducation traditionnelle, qu'elle ne doit pas répondre à son amour sous peine d'avoir 'à essuyer ses bizarreries, ses caprices, ses hauteurs, ses infidélités, tous les tourments enfin qu'un amour malheureux traîne à sa suite [...] une passion est toujours un malheur pour une femme' (35), soit de manière subie et paralysante - c'est le cas généralement d'un Meilcour:

Outré d'une aussi impudente hypocrisie [...] d'abord je ne répondis rien: je ne pouvais marquer à Mme de Lursay tout le mépris qu'elle m'inspirait, et lui répéter les discours sur lequel il était fondé, sans qu'elle se crût obligée de me rendre toute la bonne opinion que j'avais eue d'elle, et je me mettais par là, peut-être, dans l'impossibilité d'en triompher jamais (125).

La prudence dictée par le souci de préserver l'avenir, en dépit d'une réprobation morale, interdit à la spontanéité de s'exprimer sans retenue ni contrainte et scinde le personnage qui en devient aphasique: '[j]e ne savais que lui dire'(126). L'incapacité à vivre le présent par une considération craintive de l'avenir s'accompagne de l'impuissance inverse à réaliser son propre avenir par soumission au présent: Meilcour, à la fin de la première partie, est prêt à se venger de Lursay après avoir entendu les anecdotes rapportées par Versac sur son compte. Crébillon s'amuse à le faire 'courir' chez Mme de Lursay dans cette intention pour lui marquer 'le mépris le plus outrageant' (114). La deuxième partie commence sur le rappel de ce projet qui d'une part est devenu une résolution, et de l'autre précise qu'il n'est plus prévu de la 'mortifier seulement pour le moment', mais de lui faire 'une de ces scènes éclatantes qui perdent une femme à jamais' (115). C'est au sommet de cette exaltante considération d'un projet qui le ferait 'débuter brillamment dans le monde' (115), que le recul s'enclenche. Meilcour découvre trois raisons pour ne pas poursuivre son idée: son caractère lymphatique (le projet est difficile à exécuter); son éducation morale ('je n'étais pas assez mal né pour qu'il 
me restât longtemps dans l'esprit'); enfin sa personnalité sociale ('il me fallait un mérite supérieur, ou du moins une réputation établie comme celle de Versac'). Lancé dans la vengeance à la manière d'un héros tragique, ${ }^{16}$ Meilcour bat en retraite à la manière d'un Tartuffe bouffon: '[j]'en revins donc à prendre avec moi d'autres arrangements ${ }^{17}$ plus faciles' (115). Il a beau se donner de grands airs ('comblé de joie d'avoir pu trouver une si belle vengeance et déterminé à la remplir à l'instant même'), Crébillon lui arrache progressivement l'énergie indispensable à son projet. Il commence par un 'air décontenancé', ensuite sa jeunesse le pousse à 'oublier' combien la conquête de Mme de Lursay était peu flatteuse ('à l'âge que j'avais alors, le préjugé ne tient pas contre l'occasion' [122]). La scène s'achève sur la déconfiture du personnage qui sort du salon 'honteux d'une entreprise qui avait si mal réussi' (123). Entre temps, il avait pourtant montré une détermination intrépide conforme à son projet révisé à la baisse, mais le romancier ne lui laisse aucune chance en lui attribuant une maladresse indélicate et offensante. Ce type de dépossession de soi par incapacité à s'engager dans un projet est constant dans le roman. Il ne concerne pas seulement des projets immoraux; il s'attache aussi à des projets plus honnêtes. Ainsi, Meilcour, près de quitter Mlle de Théville et de perdre 'alors l'unique bien de sa vie', n'ose pas 'hasarder' une prière auprès de sa mère de lui permettre de voir la jeune fille (155). Aux freins d'un caractère pusillanime, le romancier se plaît à ajouter à la fin de cette séquence un obstacle externe sous la forme d'une intrusion d'un tiers dans l'intimité du personnage. Meilcour a enfin réussi à présenter sa demande à Mme de Théville mais Mme de Lursay parvient à s'interposer sans que le jeune homme, 'charmé de l'espérance de voir le lendemain ce qu'il adorait', ne se méfie le moins du monde. Ainsi le personnage est trahi tour à tour par son esprit qui le décourage et par son coeur qui l'aveugle: la considération de l'avenir ou 'retient' ou 'charme' mais ne mène à rien d'autre qu'à l'échec.

\section{La durée personnelle ou le temps intérieur}

Le même phénomène affecte la durée intérieure des personnages dans leur rapport au passé comme dans leur rapport à l'avenir. Le modèle moderne contraste, par sa fragilité ou son inconsistance, avec la vie à l'ancienne.

Ainsi la mère de Meilcour, toute mondaine qu'elle est, ne tourne pas le dos à son passé au moment de son veuvage: elle symbolise la permanence d'un engagement, en compensant par sa 'tendresse' l'accident temporel qu'introduit la mort du père dans l'histoire de la famille. Le cœur se substitue au corps manquant; la rupture de la durée familiale 
est immédiatement effacée au profit de la permanence par ce projet maternel de 'me tenir lieu de tout ce que j'avais perdu en perdant mon père' (15). Le temps individuel de la mère n'est évoqué que pour être sur-le-champ écarté: '[s]a tendresse pour moi ne lui fit envisager d'autre plaisir que celui de m'élever'; la mère 'belle, jeune et riche', ne répond pas aux possibles qui se présentent à elle 'restée veuve' pourtant 'dans un âge où il n'est pas d'engagements qu'elle ne pût former' (ibid.). Son temps est donc celui de la répétition, de la fidélité institutionnelle à la famille, au maintien des charges et des devoirs. Il s'oppose à celui de la femme 'coquette' et de la femme 'galante'; en elle, le temps social et le temps personnel coïncident; la mère est ce qu' elle doit être; sa vie adopte le sens de ses responsabilités; mutatis mutandis, il en va de même de la mère $\mathrm{d}^{\prime}$ Hortense et de la Dame inconnue.

Le fils vit le temps de façon toute contraire; soit il éprouve un 'ennui intérieur' qui le prive de sens: '[j]e sentis que tout manquait à mon coeur [...]. Je fus quelque temps sans comprendre la sorte de volupté qui m'était nécessaire;' soit il déborde d'une surabondance d'occasions qui le privent tout autant de direction: '[l]es sentiments que l'une m'inspirait étaient détruits, le moment d'après, par ceux qu'une autre faisait naître' (17), si bien qu'il ne connaît que deux sortes de temporalité: une temporalité désorientée, ou une temporalité disloquée dont les effets se conjuguent à ceux de la vie sociale qui l'entoure. Le temps intérieur de Meilcour ne cesse de se diviser en instants contraires qui s'additionnent: ' $j$ 'employai ma nuit presque tout entière, tantôt à rêver aux moyens de rendre Mme de Lursay sensible, tantôt à m'encourager à ne plus penser à elle' (42); ou se superposent: '[j]e m'endormis en donnant des désirs à Mme de Lursay, et je ne sais quel sentiment plus délicat à mon inconnue' (68); ou s'annulent: '[s]a conquête à laquelle il y avait si peu de temps j'attachais mon bonheur, ne me paraissait plus digne de me fixer' (54). Parfois c'est l'épaisseur temporelle elle-même qui brise la durée personnelle: '[j]e me ressouvins en chemin, raconte Meilcour prêt à aller aux Tuileries après une conversation avec Germeuil qui a parlé d'Hortense, du rendez-vous que m'avait donné Mme de Lursay, mais [...] je ne me sentais pas assez de tranquillité dans l'esprit pour le soutenir' (69). Une première figure du temps personnel se dégage ainsi: une unité de temps se disloque sous l'effet d'une bipolarité sentimentale ni maîtrisée ni hiérarchisée: '[a]u milieu des idées flatteuses que je me formais sur ce rendez-vous [avec Mme de Lursay]: Ah! m'écriai-je, si c'était mon inconnue qui me l'eût donné!' (67).

Liée à la dislocation, une autre figure apparaît: celle d'une temporalité mobile, instable, presque fluide qui oblige le mémorialiste à construire des paragraphes dont chaque phrase détaille tout à la fois un moment divisé et un moment distingué (différent) du précédent: 
En me pénétrant du plaisir d'être si près d'une personne pour qui je sentais tant d'amour, je ne me consolais point de ne pouvoir pas l'entretenir. Son visage n'était pas tourné absolument de mon côté, mais j'en découvrais assez pour ne pas perdre tous ses charmes. La situation où elle était l'empêchait de me voir, et m'en faisait par là moins regretter ce que j'y perdais. (71)

Chaque phrase est scindée par une négation absolue ou relative de l'un de ses membres (point, mais, moins). Chaque phrase nuance le sentiment exprimé par celle qui la précède: l'inconsolé de la première se contente de moins dans la seconde (il passe de l'absolu au relatif) et trouve une satisfaction dans la troisième dont la synthèse pragmatique contredirait presque le sentiment initialement énoncé. Dans cet exemple, le temps est toutefois constructeur d'un sens; il arrive au contraire qu'il défasse tout sens posé antérieurement, révélant de la sorte une autre de ses figures: la fragilité.

Le temps est fragile par sa réversibilité. D'un moment à l'autre, la conscience des personnages se modifie sous la pression des circonstances vécues, soit par négation d'un progrès: '[l]a conversation que je venais d'avoir avec Mme de Lursay me faisait réfléchir sur mon inconnue avec plus de froideur qu'auparavant' (68); soit par annulation d'une modification et retour ${ }^{18}$ à un état antérieur: Mme de Lursay 'ne fut pas plutôt rassurée sur mon coeur qu'elle reprit à peu de chose près son ancien système' (65). Parfois, un gain est suivi d'une perte: '[c]ette idée et son empressement à voler vers moi, me causèrent un instant de plaisir. Je la regardai fixement, mais, mon attention la gênant sans doute, elle baissa les yeux en rougissant et me quitta. Je retombai dans ma première douleur' (182); parfois un projet est renversé: '[j]'étais devenu stupide et ce qu'il y a de plus singulier, $c^{\prime}$ est que tout cela se passait dans mon coeur pour une femme à qui, le moment d'auparavant, j'aurais dit avec joie: "Rompons, ne nous soyons plus rien l'un à l'autre," dont le changement m'était nécessaire, et dont la seule idée m'était insupportable' (258); tantôt un refus récent est nié: '[e]lle me quitta, et me laissa d'autant plus piqué que je croyais m'être compromis en la priant d'une chose qu'un moment auparavant $j$ 'avais refusée d'elle, et que j'avais vainement abaissé mon orgueil' (225); tantôt un instant d'exaltation amoureuse cède la place à la poursuite linéaire d'un engagement antérieur: '[q]uelques chagrins que j'en dusse prévoir, j'imaginais plus de plaisir à être malheureux par mon inconnue, qu'heureux auprès de Mme de Lursay' (81), proclame héroïquement Meilcour en fin d'après-midi aux Tuileries, avant de reconnaître plus tard dans la soirée la puissance d'attraction de Lursay: 
Je ne pus la regarder sans une sorte de complaisance que je n'avais jamais eue pour elle; nos yeux se rencontrèrent; la langueur que je trouvai dans les siens fit passer jusque dans mon coeur le mouvement que ses charmes avaient fait naître et dont le trouble semblait s'accroître à chaque instant [...] . Elle profita de tout l'amour que j'avais pour mon inconnue. (83)

La dernière formule exprime à merveille la perméabilité sentimentale d'un personnage qui au contact de la réalité, en présence de l'autre, perd lui-même toute consistance, toute réalité - oserons-nous dire - et revient sur ses décisions, ses projets, ou ses ambitions. La présence sensorielle affecte le présent du personnage de façon perceptible; parfois aussi de façon muette, inconsciente même: ' $[\mathrm{m}]$ on coeur était troublé par la colère, mais tranquille du côté de l'amour'. Meilcour se croit donc maître de lui; or il n'en est rien. Le paragraphe se clôt sur une concession, un recul: ' $[\mathrm{d}] \mathrm{u}$ moins tout entier à la haine que $\mathrm{m}$ 'inspirait Mme de Lursay, n'eus-je pas lieu de me douter que je la trouvais belle' (262). Crébillon excelle dans l'invention narrative de ces moments clivés.

C'est pourquoi il convient de suggérer ici un dernier aspect du temps dans les Égarements, celui de la rédaction même des Mémoires.

A tous ces temps personnels et sociaux ${ }^{19}$ qui ne cessent de se contrarier dans leur succession ou leur superposition, il faudrait ajouter, en effet, le jeu du mémorialiste qui utilise en général le présent de l'écriture pour renforcer la séparation, l'incommunicabilité des personnages. Il aime procéder à des reconstitutions vraisemblables de la vie intérieure d'autrui, mais c'est en général pour établir une signification négative; soit une erreur d'interprétation, comme Versac persuadé qu'Hortense lui résiste en raison de la présence de Mme de Théville (139); soit une ignorance, comme Senanges à propos de Meilcour qu'elle imagine informé des moeurs à la mode (152); soit un violent dépit, comme Lursay 'infiniment mécontente' de la conduite mortifiante de son jeune amant (170); soit le bilan négatif d'une situation, par la même pour qui ‘le mal était certain et le remède difficile' (183); soit enfin une abstention tactique (262). Ainsi le mémorialiste n'atténue pas les effets de séparation, d'isolement, d'enfermement intérieur, d'aveuglement, qui donnent au temps de son récit ses caractéristiques ironiques et comiques: le temps disloque, égare et sépare.

\section{Un roman paradoxal de la défaite du temps}

Le roman ne parvient plus à restituer le tableau d'une humanité unifiée, d'un être humain cohérent, d'une société stable; il goûte plus d'une fois à la tentation de se transformer en traité de morale fustigeant par des formules de vérité générale énoncées au présent d'éternité le temps 
'fabuleux' de sa jeunesse ${ }^{20}$ comme s'il lui fallait abîmer et détruire de façon définitive son propre passé pour s'interdire toute communication positive avec lui, pour enlever au lecteur toute possibilité d'adhésion, de communion avec ce temps. Le roman nous prive de toute reconstruction mythique d'un temps séduisant, beau ou grandiose: il se métamorphose en exercice de dégrisement. Le temps ne fabrique que solitude.$^{21} \mathrm{~L}$ 'entente est donc une illusion cruellement soulignée un jour par le mémorialiste: '[e]lle me quitta en soupirant, et très persuadée que nous commencions enfin à nous entendre, quoique au fond il n'y eût qu'elle qui se comprît' (66). Aucun temps vécu ne vaut la moindre chandelle d'une reconstitution esthétiquement attirante. Le temps ne possède plus la majestueuse force esthétique et morale de la tragédie; il se contente désormais de ruiner toute prétention à la valeur; il fait des personnages en scène des orphelins du sens. C'est ce que Crébillon, s'inspirant de la Comédie, appelait 'le tableau de la vie' (10).

\section{JACQUES WAGNER}

Université Blaise-Pascal (Clermont-Ferrand)

\section{Notes}

1 Héraclite, Fragments (Paris: Calligrammes, 1987) 19.

2 Boèce, Consolation de la philosophie (Marseille: Rivages, 1989) 187.

3 Marc Fumaroli le rappelle justement dans la préface de l'ouvrage cité: 'Seule la connaissance rationnelle, dépassant ces vues infléchies par le corps et le milieu terrestres, est en mesure de s'élever à la vision de l'universel, et de comprendre l'ordre cosmique voilé. La connaissance prisonnière du temps, trompée par la succession fragmentée du passé, du présent et du futur immédiat, est d'un ordre inférieur et illusoire; il est une autre connaissance qui adopte sur le temps le point de vue de l'éternité, et remplace la fragmentation des instants par une vision spatiale et unifiante, où tout se rassemble et trouve la plénitude de son sens' (ibid. 37).

4 Pascal dramatise le thème du flux universel: ' $C$ 'est une chose horrible de sentir s'écouler tout ce qu'on possède' (Pensées [Paris: Seuil / Lafuma, 1963] 597); La Rochefoucauld, en une seule maxime, parvient à ridiculiser les deux aspects inverses du temps humain: 'Il est aussi ordinaire de voir changer les goûts qu'il est extraordinaire de voir changer les inclinations' (Maximes [Paris:

Larousse-Roustan, 1900] 70); le temps valorisé de la culture est maltraité par l'homme; le seul temps stable sera, ironiquement, celui de la biologie, de la nature, des passions, celui qui échappe à la volonté libre de l'homme.

5 Les rêveries du promeneur solitaire (Paris: Garnier, 1960) 70. 
6 Jankélévitch a très finement et très joliment développé le contraste entre l'homme de la continuation douillette et l'homme de la futurition ouverte, 'réconcilié avec le devenir', dans L'aventure, l'ennui, le sérieux (Paris: Aubier-Montaigne, 1963) 43.

7 La lenteur, que Kundera a récemment analysée à partir de Vivant Denon, a fait partie de l'univers moral des classiques qui l'ont encensée, que ce soit Boileau:

Travaillez à loisir, quelque ordre qui vous presse,

Et ne vous piquez point d'une folle vitesse.

Un style si rapide, et qui court en rimant,

Marque moins trop d'esprit, que peu de jugement.

J'aime mieux un ruisseau qui, sur la molle arène,

Dans un pré plein de fleurs lentement se promène,

Qu'un torrent débordé qui d'un cours orageux

Roule plein de gravier sur un terrain fangeux.

Hâtez-vous lentement, et sans perdre courage,

Vingt fois sur le métier remettez votre ouvrage (Art poétique, I, 162-171);

ou La Bruyère: 'Il n'y a pas de chemin trop long à qui marche lentement et sans se presser' (Caractères, XII, §108).

9 Crébillon, Claude, Prosper Jolyot de, Les égarements du cour et de l'esprit (Paris: Les Belles Lettres, 1996) 19. Désormois dans le corps du texte.

9 Il faut noter la différence d'application de ce phénomène selon qu'il s'agit d'un homme ou d'une femme: pour l'un, on évoque soit le manque d'expérience de la jeunesse: 'Il est un âge où tout plaît, c'est un malheur' (199); soit la fougue séductrice: 'Rassurez-moi contre tout ce que j'ai à craindre de votre âge' (86).

10 Versac ne lâche prise à aucun moment de la soirée car il n'est venu chez son amie 'que pour se donner le plaisir de la mortifier' (143).

11 Les raisons de cette opacité sont multiples: soit le code de déchiffrage manque à l'observateur, soit les états d'âme (mouvements, passions, inquiétudes, doutes) $s^{\prime}$ interposent et paralysent ou corrompent le jugement. Il y a là comme un reste de cartésianisme diffus dans le texte de Crébillon.

12 La bonne comtesse, pénétrée de la plus auguste douleur, ne se montre plus en société depuis longtemps, selon Versac qui s'exclame: 'Mais c'est qu'on ne la voit plus. J'en suis dans une douleur amère, dans la plus terrible affliction' (110). Mme de Lursay 'fit des reproches à son amie (Mme de Théville) de ce qu'elle avait été longtemps sans la voir' (127). Une variante est utilisée par Senanges à propos de Lursay: 'Mais dites-moi, Reine, ce que vous devenez, qu'il n'est plus possible de vous trouver nulle part' (130).

13 Versac, décrivant les techniques de séduction mondaine, conseille de s'interdire tous les comportements agréables: 'Le trop grand désir de plaire (aux gens) suppose le besoin qu'on en a. Ils ne sont jamais plus portés à nous juger avec sévérité, que lorsqu'ils nous voient chercher servilement à nous les rendre favorables. [...] Cette crainte de lui déplaire, même en le flattant, ne nous le gagne pas. L'hommage que nous lui rendons l'enhardit à nous trouver des défauts, sur lesquels, sans nos ménagements pour lui, il n'aurait peut-être jamais osé porter ses yeux. Il est vrai qu'il veut bien s'y prêter, mais la bonté avec laquelle il les excuse est une injure pour nous, que plus de confiance en nous-mêmes nous aurait épargnée' (238).

14 Versac le reconnaît dans une phrase alambiquée: 'Cet orgueuilleux [...] qui en blâmant nos vices, nous estime assez peu pour ne plus nous dissimuler les siens, 
se serait cru trop heureux d'obtenir de nous l'indulgence qu'il nous accorde, si nous n'avions pas cru avoir besoin de la sienne' (238).

15 Devant les appréhensions de Meilcour qui trouve la méthode bien pénible, Versac assure que 'l'on peut réduire l'art de plaire aujourd'hui à quelques préceptes assez peu étendus, et dont la pratique ne souffre aucunes difficultés' (234).

16 Cf. le 'Va, cours, vole et nous venge' de Don Diègue, qui dépeint l'ardeur des engagements dignes des vrais héros tragiques ( $L e$ Cid, I, 5, 290) est repris par Rodrigue:

Courons à la vengeance;

Et tout honteux d'avoir tant balancé,

Ne soyons plus en peine

Puisqu'aujourd'hui mon père est l'offensé,

Si l'offenseur est père de Chimène' (ibid. 350).

17 Sur ce mot, voir la note suivante; cette attitude n'est pas sans faire penser aux 'accommodements' du Tartuffe de Molière (IV, 5, 1488); dans une tonalité moins tendue, plus légère.

18 Ce mot se trouve dans la bouche de Meilcour: 'Quoique Mme de Lursay me parût enfin s'être arrangée sur ses scrupules, je ne laissais pas de craindre un de ces retours auxquels elle était sujette' (88).

19 Il serait fécond de développer le détail des oppositions systématiques établies entre temps personnel et temps collectif: échec à l'intimité désirée ou au contraire imposition d'une intimité redoutée, échec aussi à la civilité par les disputes, les humeurs qui aboutissent à des disputes.

20 Le mémorialiste prend soin de prévenir son lecteur que 'les moeurs ayant depuis ce temps-là si prodigieusement changé', il ne serait pas surpris qu'on traitât de fable aujourd'hui 'ce qu'il vient de dire sur la manière d'aimer des libertins' (20).

21 Le contraste avec un roman comme Les illustres françaises de Challe est flagrant. 